\title{
The impact of maternal prenatal and postnatal anxiety on children's emotional problems: a systematic review
}

\author{
Sarah Rees ${ }^{1} \cdot$ Susan Channon ${ }^{1} \cdot$ Cerith S. Waters $^{1}$ \\ Received: 27 September 2017 / Accepted: 22 May 2018 / Published online: 15 June 2018 \\ (c) The Author(s) 2018
}

\begin{abstract}
Maternal mental health problems during pregnancy and the postnatal period are a major public health issue. Despite evidence that symptoms of both depression and anxiety are common during pregnancy and the postpartum, the impact of maternal anxiety on the child has received relatively less attention than the impact of maternal depression. Furthermore, the evidence base for the direct impact of maternal anxiety during pregnancy and the postpartum on children's emotional outcomes lacks cohesion. The aim of this systematic review is to summarise the empirical evidence regarding the impact of maternal prenatal and postnatal anxiety on children's emotional outcomes. Overall, both maternal prenatal and postnatal anxiety have a small adverse effect on child emotional outcomes. However, the evidence appears stronger for the negative impact of prenatal anxiety. Several methodological weaknesses make conclusions problematic and replication of findings is required to improve the identification of at-risk parents and children with appropriate opportunities for intervention and prevention.
\end{abstract}

Keywords Perinatal $\cdot$ Maternal anxiety $\cdot$ Child $\cdot$ Emotion $\cdot$ Development

\section{Introduction}

Perinatal mental health refers to a woman's mental health during pregnancy and the first year after birth. This includes mental health difficulties existing before and persisting into the pregnancy, as well as mental health problems that develop for the first time, or are greatly exacerbated in the perinatal period. Depression and anxiety are the most common mental health problems during pregnancy, with approximately $12 \%$ of women experiencing depression and $13 \%$ experiencing anxiety at some point, with many women experiencing both [e.g., 1-6]. Depression and anxiety also affect $15-20 \%$ of women in the first year after birth [1, 7]. Despite this, an estimated 40-70\% of women in the UK have no access to specialist perinatal mental health services [7].

Perinatal mental health problems are a major public health issue. It is well established that maternal mental health difficulties in pregnancy have been associated with preterm labour, poor infant outcomes, and greater cognitive, behavioural, and interpersonal problems in young children

Cerith S. Waters

waterscs@cardiff.ac.uk

1 Cardiff University, Cardiff, Wales, UK
$[6,8]$. Perinatal depression, anxiety, and psychosis carry a total long-term cost to society (including health and social care use, productivity losses, infant death, emotional problems, and special educational needs) of an estimated $£ 8.1$ billion for each 1-year cohort of births in the UK [7], with $72 \%$ of this cost relating to the adverse impacts on the child rather than the mother [7]. Perinatal anxiety (when it exists alone and is not co-morbid with depression) costs an estimated $£ 35,000$ per mother-child dyad of which $£ 21,000$ relates to the mother and $£ 14,000$ to the child [7].

The research literature has predominantly focused on postnatal depression [9], which is associated with reduced maternal sensitivity to the child and adverse offspring cognitive, behavioural, and emotional outcomes, particularly for boys [10-13]. Although there is evidence that symptoms of both depression and anxiety are common prenatally, less attention has been paid to the direct impact of symptoms of anxiety occurring during and after pregnancy [3]. In a systematic review of the impact of postnatal maternal anxiety on child development [8], the outcomes were categorised into three domains: somatic, developmental, and psychological. The strongest evidence for an adverse effect of postnatal maternal anxiety exposure was on offspring somatic and psychological outcomes (in which emotional outcomes were embedded) with the evidence for an effect of 
postnatal maternal anxiety on child developmental outcomes (developmental milestones and cognitive delay) found to be inconclusive.

Research data indicate that greater than typical elevations in maternal perinatal stress, anxiety and depressive symptoms are associated with a wide range of adverse cognitive, behavioural, and neurophysiological offspring outcomes [14]. A dominant hypothesis is that there are prenatal programming effects for psychopathology, a process known as 'fetal programming'. However, the evidence base for the direct impact of perinatal maternal anxiety on children's emotional problems lacks cohesion, often embedding such findings within broader child developmental outcomes [15]. This poses a challenge to those who wish to draw upon research in this area to guide clinical practice and further research developments. Therefore, the aim of this review is to systematically summarise studies which measure the impact of maternal perinatal anxiety on child emotional problems to enable future practitioners and researchers to draw conclusions from the findings.

\section{Methods}

The guidance outlined in the Preferred Reporting Items for Systematic Review and Meta-analysis Protocols [PRISMAP: 16, 17] was followed.

\section{Search procedures}

Articles published between 1900 through January 31st 2017 were systematically identified through 11 electronic databases (PsycInfo, PsychArticles, Pub Med, Medline, BIOSIS citation index, BIOSIS previews, the Science Citation Index, Embase, SCOPUS, Journal citation reports, and Web of Science). Search terms included a combination of databasespecific index terms (anxiety, perinatal period, emotional development, and behaviour problems) and individual terms located in the title or abstract (maternal OR mother*, prenatal OR perinatal OR postnatal OR postpartum OR puerperal, child* OR bab* OR infant*, behaviour* OR behavior*, emotion*). The behaviour search terms were used to ensure the search captured emotional outcomes that are embedded within behavioural measures.

\section{Inclusion/exclusion criteria}

All studies that prospectively examined the impact of maternal anxiety in the prenatal and postnatal period on children's emotional outcomes were considered for inclusion. The database search was restricted to human research articles, written in English, and published in peer-reviewed journals. Studies that did not assess maternal anxiety during the perinatal period, or where anxiety could not be distinguished from other measures (e.g., 'psychological distress'; a composite variable combining maternal anxiety and depression) were excluded. Articles that focused on maternal effects and not child outcomes or did not assess child emotional problems were also excluded. The titles and abstracts of all studies identified were screened, with those meeting the inclusion criteria selected for full-text evaluation. Reference lists of identified papers were examined, and the bibliographies of key researchers, defined as the authors of the 14 studies that met the inclusion criteria, were reviewed. Related publications were searched using the investigators name(s)/study names and principle investigators were contacted. The comorbidity of perinatal anxiety and depression is acknowledged, but given that less attention has been paid to maternal anxiety, the current systematic review concentrates primarily on the direct impact of perinatal anxiety on children's emotional problems.

There is variability within the developmental literature as to how emotional problems are defined with particular terminology often linked to specific scales. For example, studies which measure emotional outcomes using the Child Behaviour Checklist (CBCL) refer to 'internalizing' difficulties which represents a composite score of the emotionally reactive, anxious/depressed, somatic complaints, and withdrawn subscales. Other studies refer to 'emotional symptoms' (measured by the Strengths and Difficulties Questionnaire; (SDQ) or 'social-emotional competence' (e.g., self-regulation, compliance, and interaction with people), measured using the Ages \& Stages Questionnaire-social-emotional; (ASQ: SE). Clinical diagnoses are also used for older children. Therefore, for the purpose of the current review, the term 'emotional problems' will be used to encompass all definitions used within the reviewed studies.

Search results were uploaded to the systematic review software Covidence [18]. Articles from all searches were combined and duplicates removed. A second reviewer independently screened full-text articles and any conflicts were discussed and resolved. A third reviewer was available to discuss screening outcomes in instances where discrepancies and uncertainties between the two reviewers were not resolved. A PRISMA flow diagram reporting the final numbers is shown in Fig. 1.

\section{Search results}

Excluding duplicates, 1,186 studies were identified through database searches. Evaluation of the title and abstracts according to the exclusion criteria decreased the articles from 1186 to 58 . Due to prior steps being conservative, several articles that did not meet the review criteria were retained for full-text review, because titles and abstracts were not specific enough to judge for inclusion. 
Fig. 1 Flow diagram of included and excluded studies
1413 Articles Identified

PsycInfo (Ovid) - $(\mathrm{n}=886)$

PubMed - $(\mathrm{n}=198)$

Web of Science $-(n=329)$

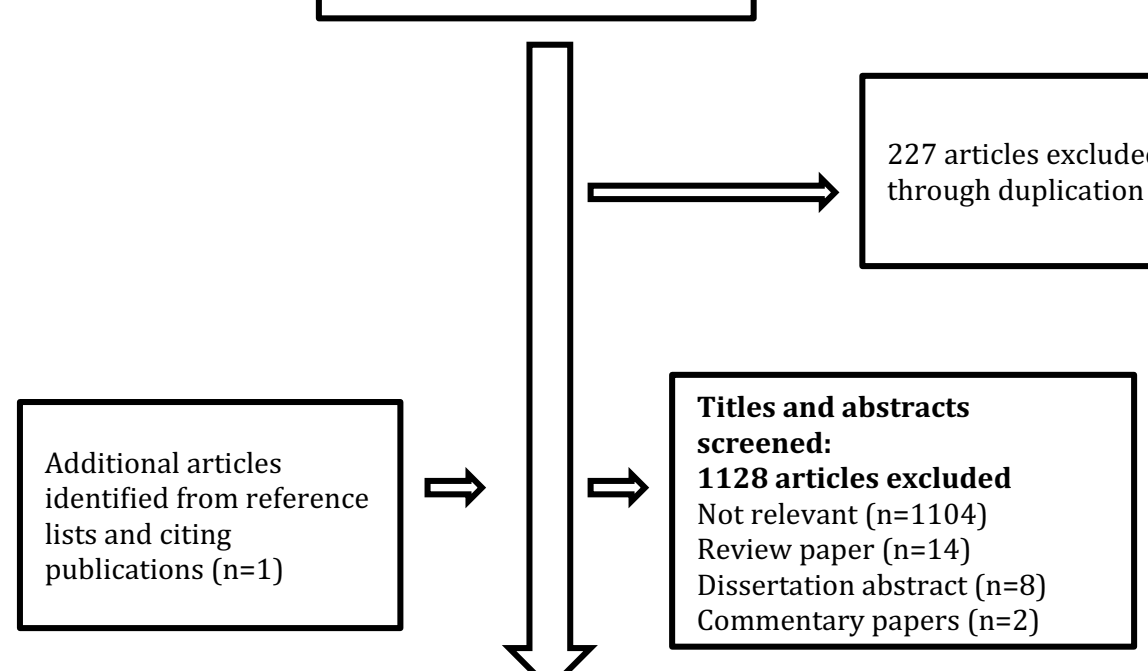

\section{9 full text papers reviewed}

All screened as per inclusion and exclusion criteria

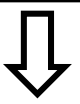

\section{5 articles excluded}

Study does not measure or report direct impact of maternal anxiety measured during perinatal period $(n=14)$

Study does not measure child emotional outcome $(n=29)$

Access to full text not available $(n=2)$

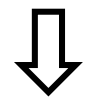

14 articles meet inclusion criteria
Evaluation of the articles' text reduced the 58 studies to 13. One additional publication was identified through the examination of the reference lists. Fourteen articles were excluded, because studies did not measure or report the direct impact of maternal anxiety measured during the perinatal period. Twenty-nine articles were excluded, because studies did not measure child emotional problems and the full text of two articles could not be accessed. 
After applying these criteria 14 studies remained, which were published between 2002 and January 2017.

\section{Assessment of the quality of evidence}

The Critical Appraisal Skills Programme (CASP) guidance, recommended for reviewing observational research, was used to aid the appraisal of the included studies. The framework provides an appraisal tool for cohort studies across 12 quality domains with a series of prompt questions for each domain. In each domain, a response of 'yes', 'no', or 'can't tell' is given. To aid the comparison of quality across studies, a score of two was given for 'yes' a score of one was given for 'can't tell' (indicating mixed achievement of the domain) and a score of zero was given to 'no'. Scores across domains were then summed to give a maximum quality score of 12 . The total quality score for each study is included in Table 1 and a summary of the quality scores for each paper reviewed can be found in Table 2.

A summary of the effect sizes for significant findings pertaining to the relationship between prenatal and/or postnatal maternal anxiety and children's emotional outcomes are reported in Table 3. If a study did not report an effect size, but it was possible to calculate an effect size based on the information provided in the published manuscript, post hoc effect sizes were computed for studies reporting significant findings. Only effect size estimates that adjusted for correlated risk factors (e.g., the effect of prenatal anxiety on child emotional problems after controlling for the impact of postnatal anxiety and depression) are reported in Table 3 . When it was not possible to extract an effect size, 'unobtainable' was recorded in the column. Conventional practice for effect size reporting was followed and small, medium, and large effect sizes were deemed as: Cohen's $d=0.2,0.5$, and 0.8 ; Cohen's $f^{2}=0.02,0.15$, and 0.35 ; and odds ratios $=1.5$, 2.5, and 4.3 [19-21].

\section{Results}

\section{Design}

Table 1 provides an overview of the included studies. Thirteen of 14 were population-based cohort studies and one used a nested case-control design. Thirteen studies began in pregnancy and one began in the postnatal period. All of the studies used a prospective longitudinal design. Seven studies examined separate study populations, while seven reported different analyses of two birth cohorts (five from one cohort and two from another).

\section{Participants}

All of the studies involved community-based samples of mother-child dyads. Sample sizes ranged from 71 to 7,944 mother-child pairs (median 1143). Ten studies reported mean maternal ages at pregnancy (range 26.8-31.8 years), two reported mean maternal age at assessment and two did not report details of mean maternal age. Children ranged from 14 months to 13 years. Twelve studies assessed children at one timepoint, one study assessed children at two timepoints ( 4 and 6 years) and one study assessed children at multiple timepoints $(4,7,9,11.5$, and 13 years).

\section{Maternal anxiety measures}

A number of different measures were used to evaluate maternal anxiety including self-report scales and standardised clinical interviews. The most frequently used measure was the State Trait Anxiety Inventory (STAI) [22, 23], which was used in six studies. The STAI has acceptable reliability and established validity and is one of the most widely used measures of anxiety symptoms [24]. Five studies measured symptoms of anxiety with the 8-item anxiety subscale of the Crown Crisp Experiential Index [CCEI; 25]. The CCEI is a well-validated self-report symptom scale and the anxiety subscale has frequently been used to measure anxiety in perinatal women [e.g., 3, 26]. One study used the Hopkins Symptom Checklist [27]. Three studies used self-report questionnaires that measure specific anxiety disorders and two used the Structured Clinical Interview for DSM-IV Diagnoses [28]. Four studies measured maternal anxiety only in the prenatal period, eight measured maternal anxiety both pre- and postnatally and two measured maternal postnatal anxiety only.

\section{Child emotional problems and outcome measures}

Emotional outcomes were measured using a variety of questionnaire scales and clinical interviews with assessments ranging from 14 months to 13 years. The most frequently used measure, in seven studies, was the Internalizing Scale of the CBCL [29, 30]. Four studies used maternal reports only, two studies included both maternal and paternal ratings, and four studies reported on teachers' ratings [31]. Five studies used maternal reports of children's emotional problems on the SDQ [32], an adaptation of a widely used index of psychiatric symptoms in children [33]. One study used maternal reports of children's social-emotional competence using the ASQ: SE [34]. Another study used both maternal and paternal reports of children's emotional problems using the Development and Well-Being Assessment, a standardised clinical interview that yields psychiatric diagnoses [DAWBA; 35]. Two studies measured child anxiety 


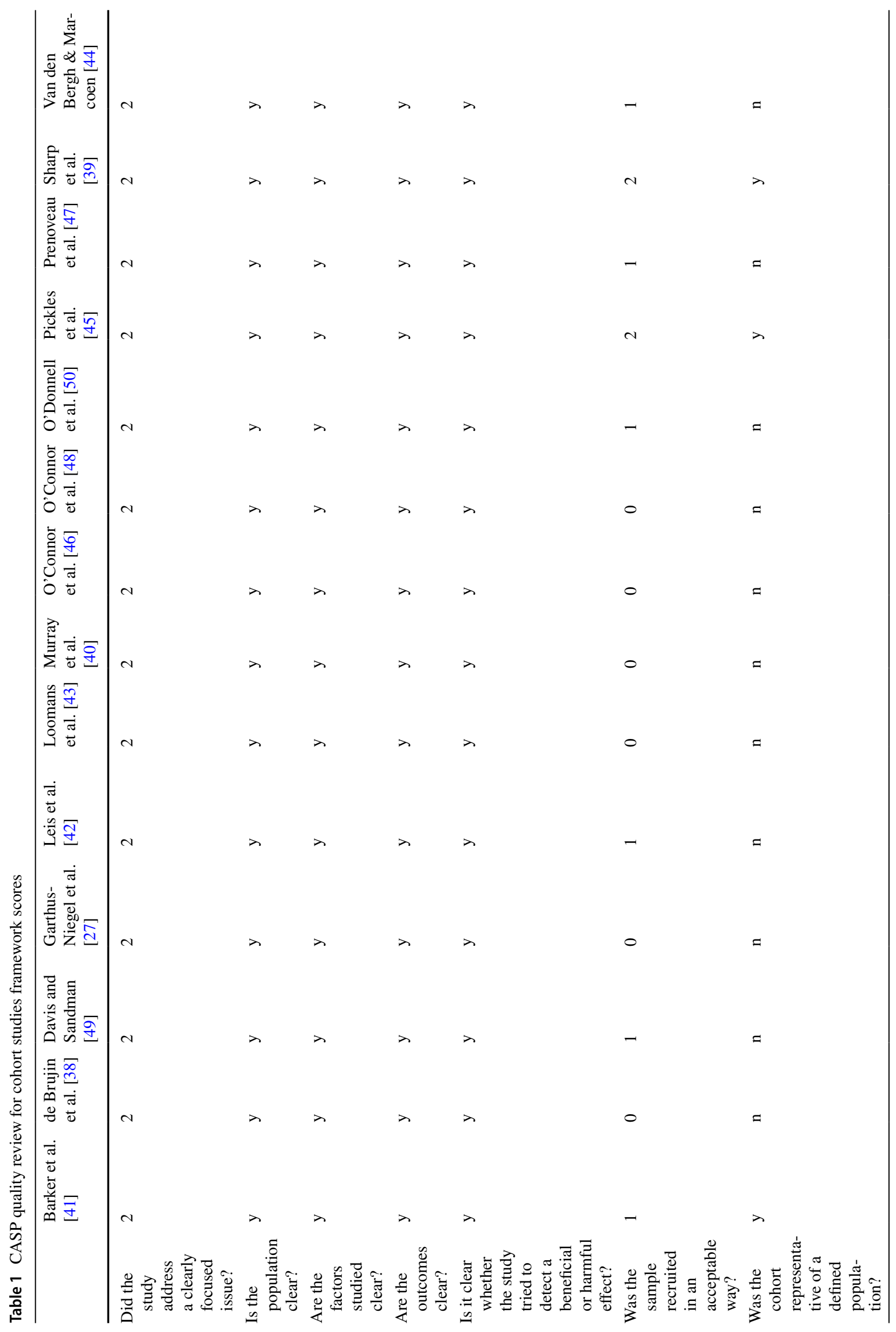




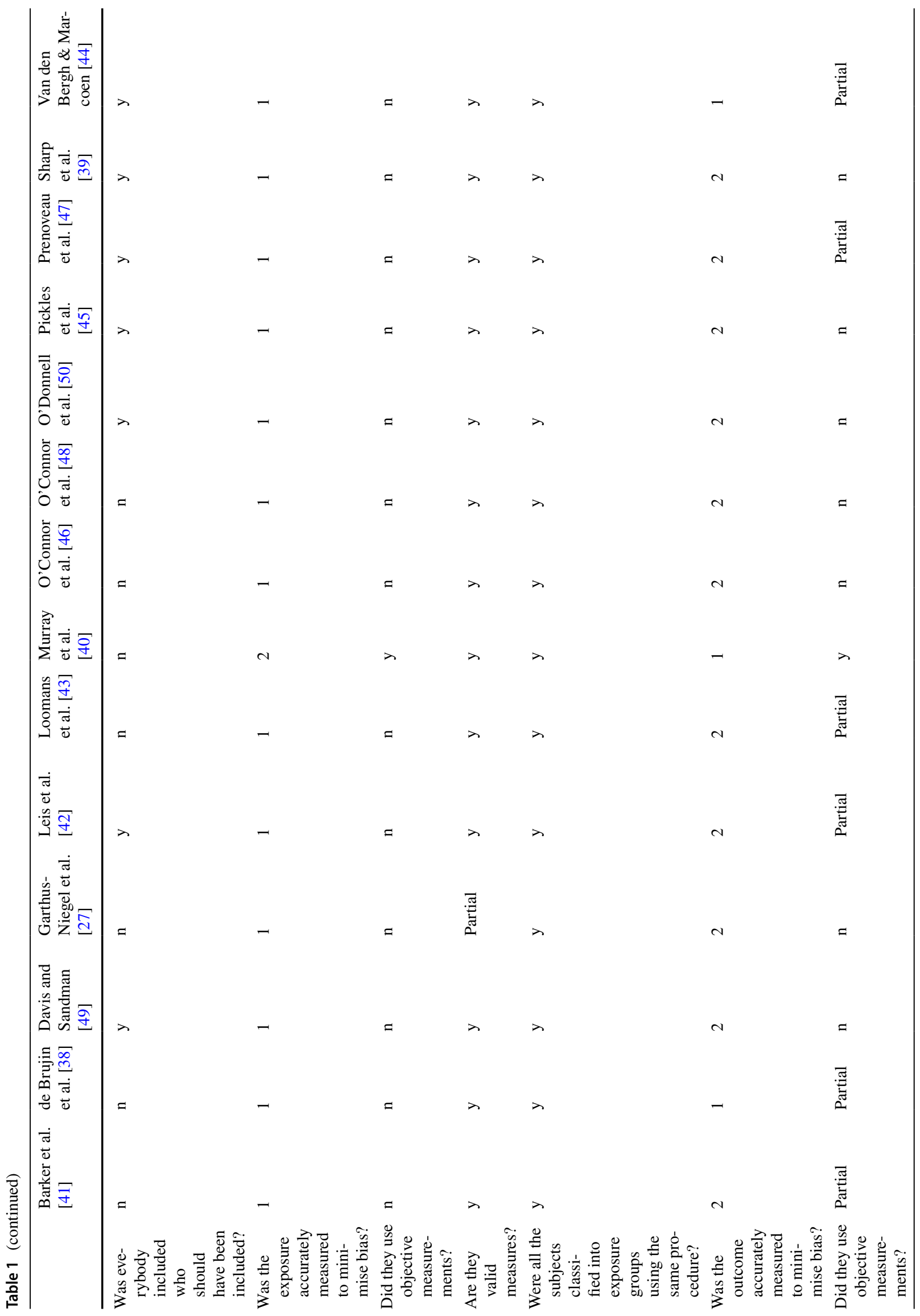




$$
\text { [ }
$$




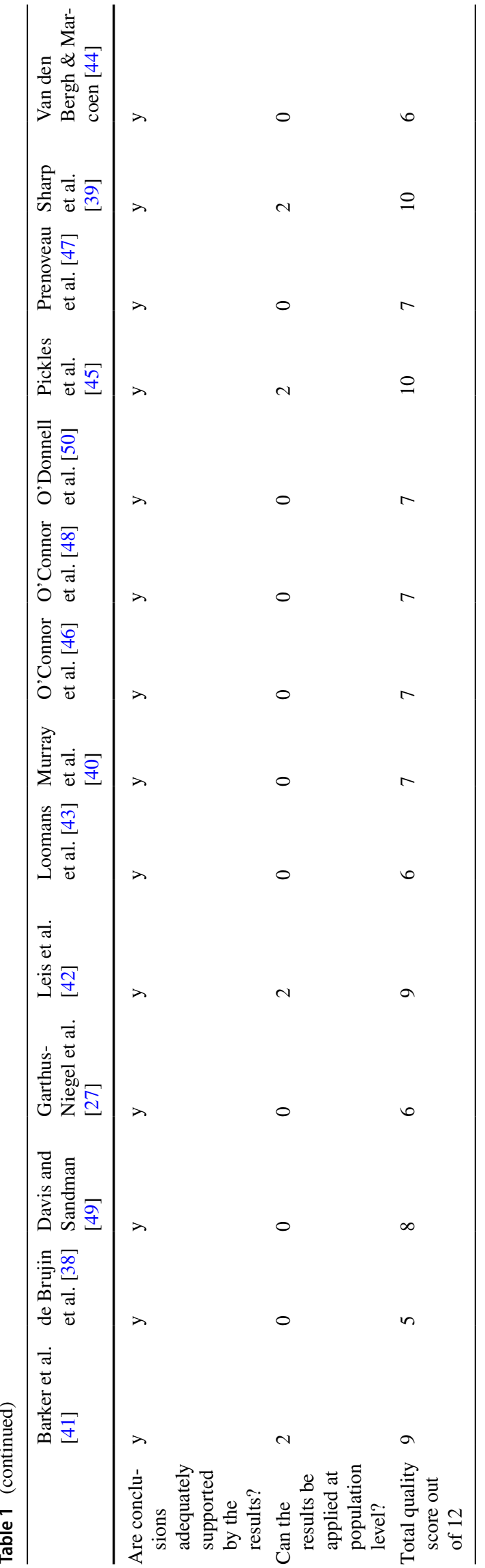

specifically alongside the CBCL: one used the Anxiety Disorder Interview Schedule, Parent Version [ADIS-P; 36] and the other used the self-report State Trait Anxiety Scale for Children [STAIC; 37].

\section{Quality of studies}

Table 2 provides an overview of the quality ratings of published articles. The quality of the studies varied from a lowest score of five [38] to a highest of 11 out of 12 [39], with higher scores indicating better quality. Six studies used a second informant (e.g., teacher rating or father rating) in addition to maternal ratings of children's emotional problems [38, 40-44]. The results of three studies were representative of the general population $[39,41,45]$.

\section{Perinatal anxiety and children's emotional problems: synthesis of the findings}

Table 3 provides an overview of the study findings. Across the identified studies, child emotional problems have been assessed at different developmental stages, with the majority of studies focusing on the early and middle childhood period. Most studies assessed maternal anxiety in the prenatal period with fewer studies assessing anxiety in the postnatal period. Thus, the findings are reported according to stages of childhood and timing of anxiety measurement (Table 3). It is noteworthy that across the 14 studies represented in Table 3, any significant association between preand/or postnatal maternal anxiety and offspring emotional problems represents a small effect size following the statistical adjustment of correlated risk factors.

\section{Early childhood emotional outcomes: the effect of prenatal anxiety}

After controlling for covariates, and when mothers reported on child outcomes, 4 studies found prenatal anxiety to be associated with elevated offspring emotional problems when children were aged between 2 and 5 years [39, 40, 43, 46]. Similarly, Pickles et al. found an initial effect of prenatal anxiety on offspring emotional problems at 3.5 years [45]. However, following adjustment for confounding factors (e.g., postnatal anxiety and depression), the association between prenatal anxiety and offspring emotional problems was no longer significant [45]. This particular study had a high-quality rating based on its strong methodology [45].

Three studies used multiple informants (mothers, fathers, and teachers) to assess offspring emotional problems in early childhood [38, 40, 43]. Murray and colleagues found that compared to children of non-anxious mothers, the offspring of socially anxious mothers were themselves more likely to 


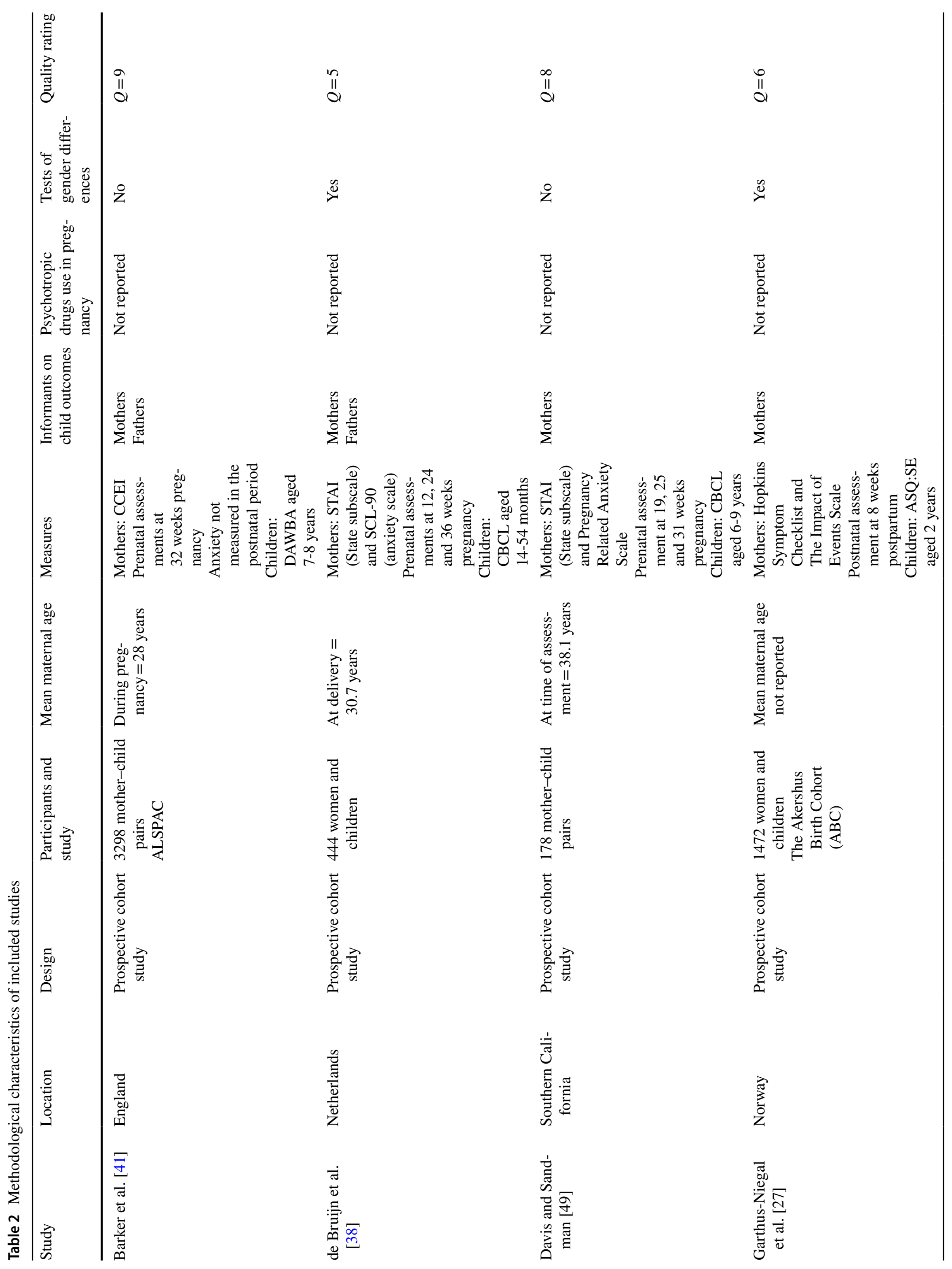




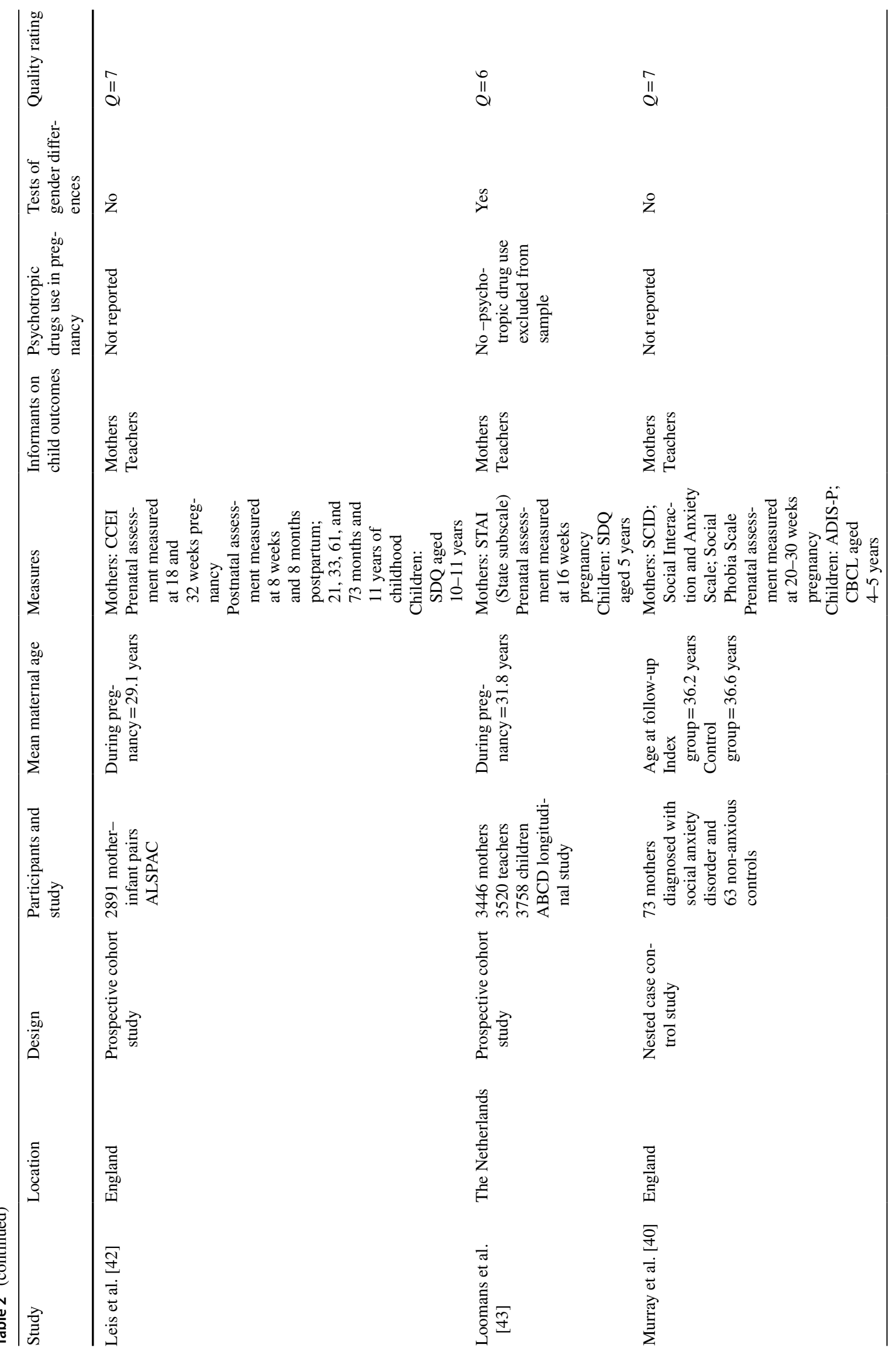




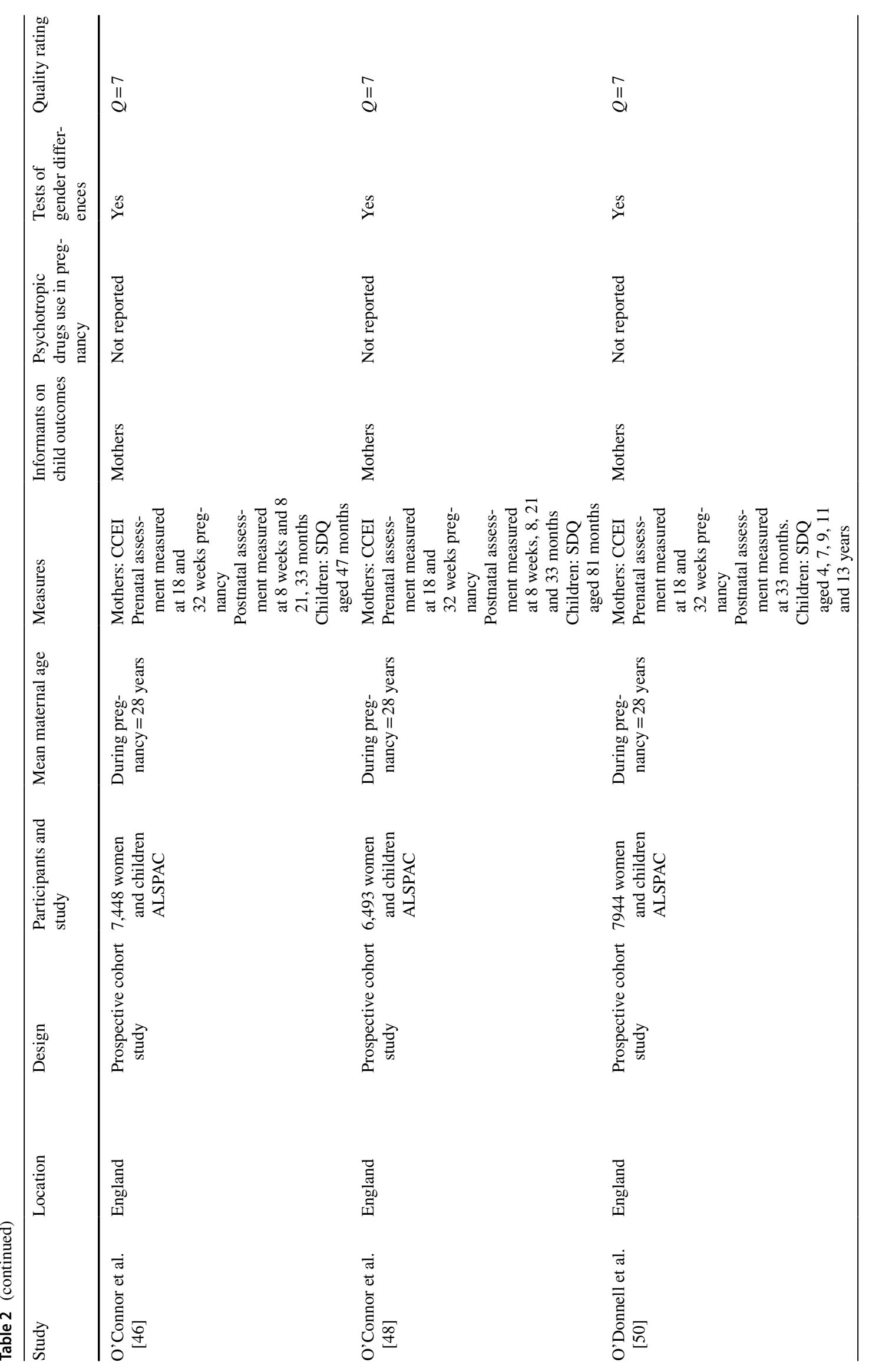




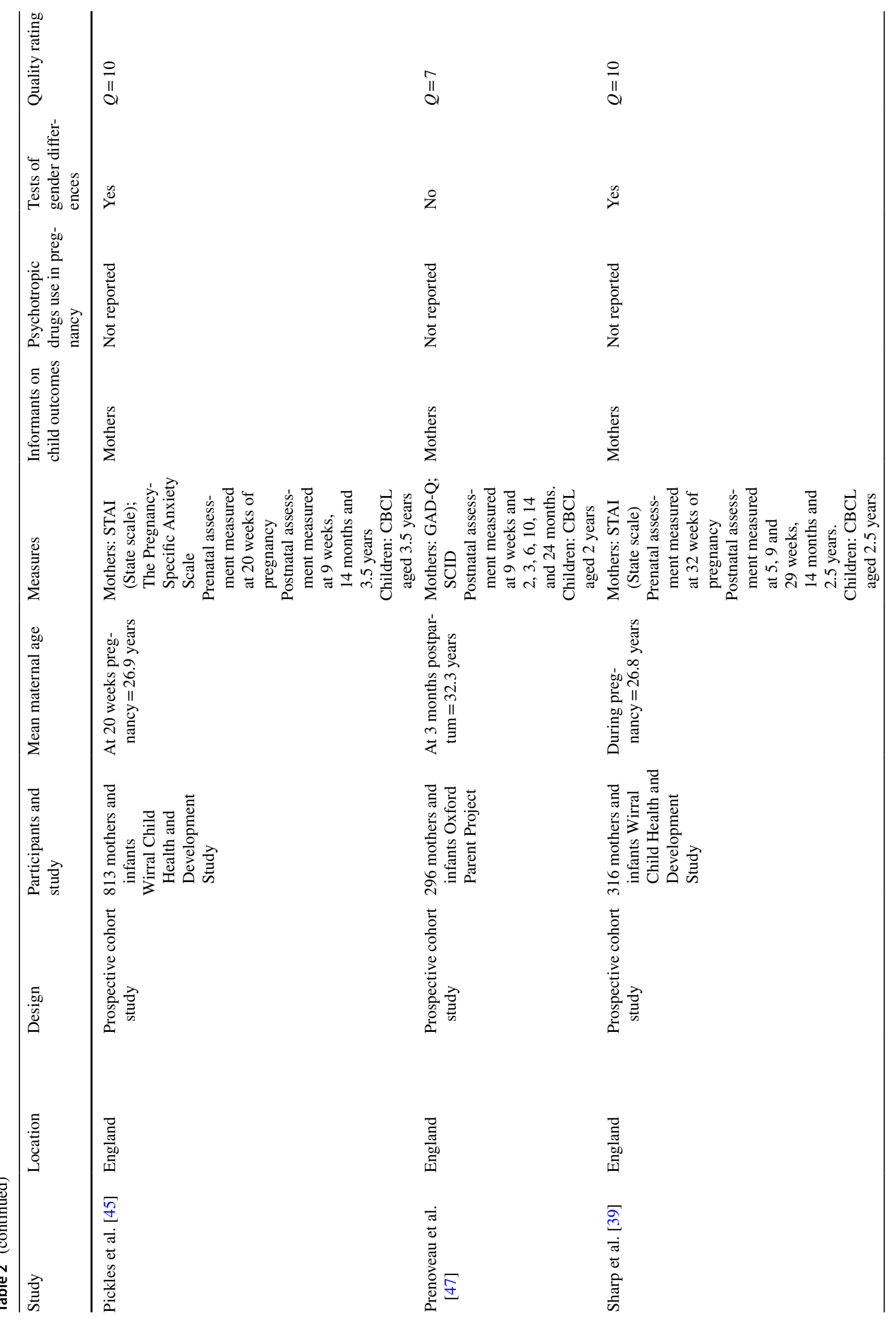




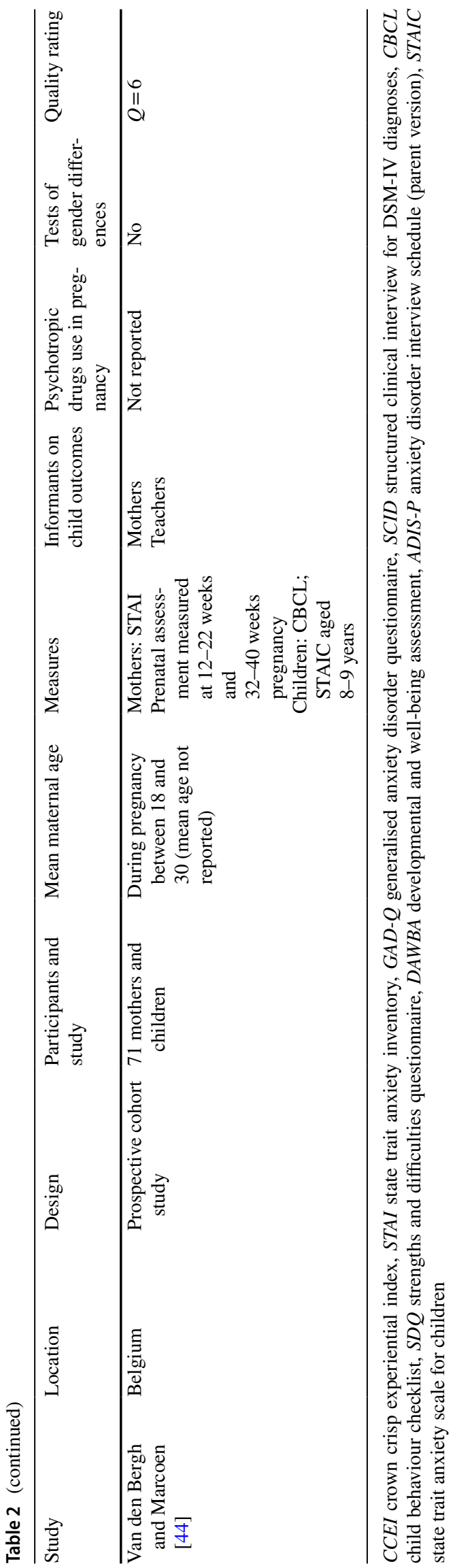

be diagnosed with social anxiety disorder [40]. However, in this study, the clinical diagnosis was based on maternal report and when teacher-rated emotional problems were used as the outcome variable, no significant relationships were observed. This is a surprising finding as one would expect children to show difficulties with social anxiety in the school environment. Thus, this finding may indicate method variance, where elevated associations between maternal anxiety and children's emotional problems are a result of mothers' reports being used for both variables. One study found a significant effect of prenatal anxiety on paternal but not maternal reports of female children's emotional problems [38]. This study scored the lowest quality rating on the CASP and a wide age range was used for the child assessment (14-54 months). Loomans and colleagues [43] found a significant positive association between prenatal anxiety and teacher-rated emotional problems; however, this association was no longer significant after controlling for maternal postnatal emotional distress (composite measure of depression, anxiety, and stress) [43].

\section{Early childhood emotional outcomes: the effect of postnatal anxiety}

In early childhood, the impact of postnatal anxiety on child emotional problems was assessed by three studies. GarthusNiegel and colleagues [27] found mothers' Post Traumatic Stress Disorder (PTSD) symptoms at 8 week postpartum predicted elevated offspring socio-emotional problems at 2 years [27]. In contrast to the PTSD symptoms, maternal reported symptoms of generalised anxiety in this study were not significantly related to offspring emotional problems after controlling for confounders [27]. O'Connor and colleagues [46] found postnatal anxiety assessed at 8 week postpartum predicted emotional problems in both boys and girls at aged 4 years [46]. Prenoveau and colleagues [47] found mothers' persistent postnatal anxiety symptoms measured at 9 weeks and 2, 3, 6, 10, 14, and 24 month postpartum predicted mothers' reports of children's emotional problems at 24 months. However, when mothers' depressive symptoms were included in the model, persistent postnatal anxiety did not independently predict maternal reported emotional problems above and beyond the impact of maternal postnatal depression.

\section{Middle childhood emotional outcomes: the effect of prenatal anxiety}

Three studies assessed how maternal prenatal anxiety impacted on offspring emotional problems in middle childhood (approximately 6-9 years of age). Barker and colleagues [41] found that prenatal anxiety, measured at 32 weeks gestation, was associated with a small increase 


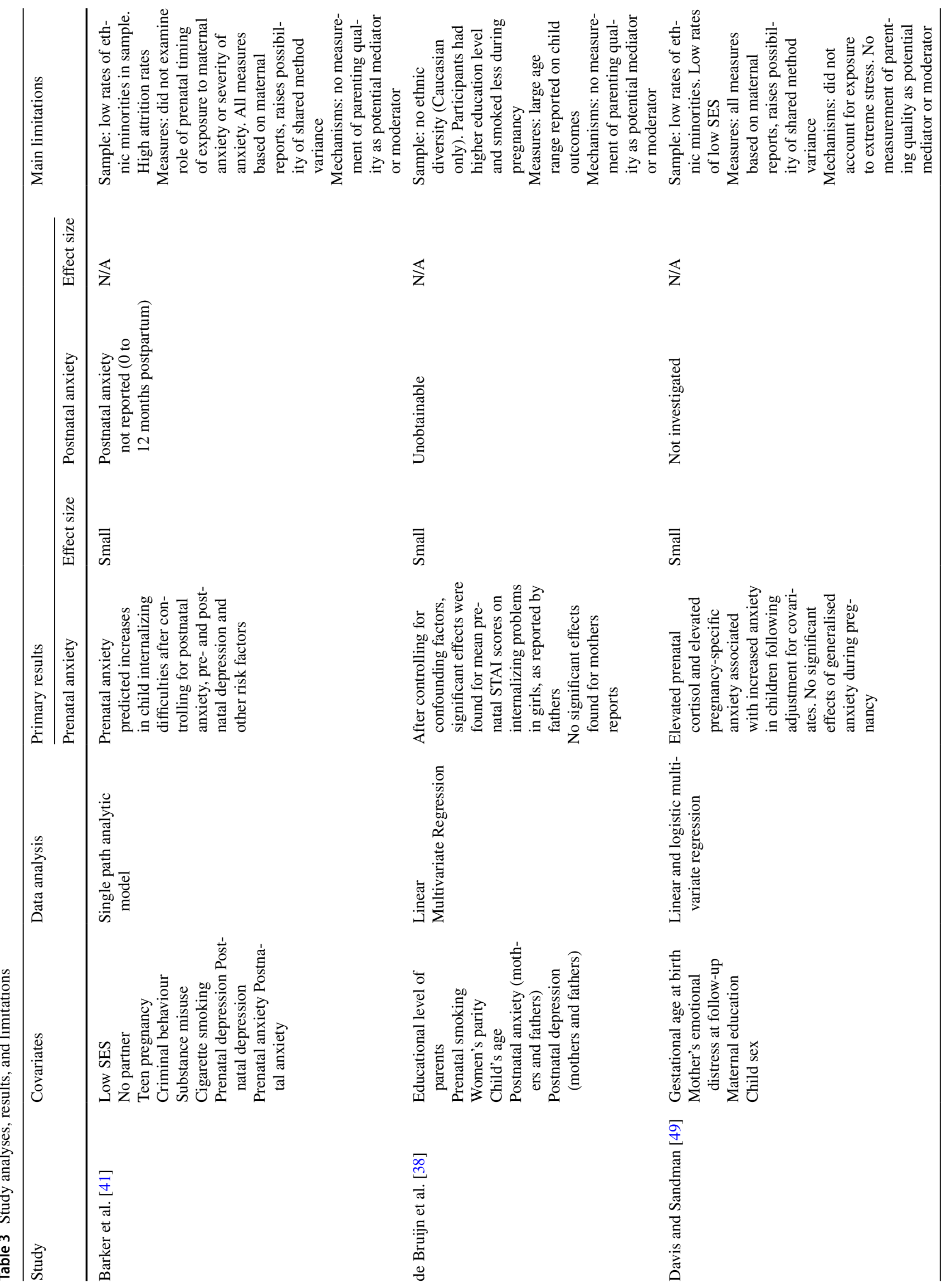




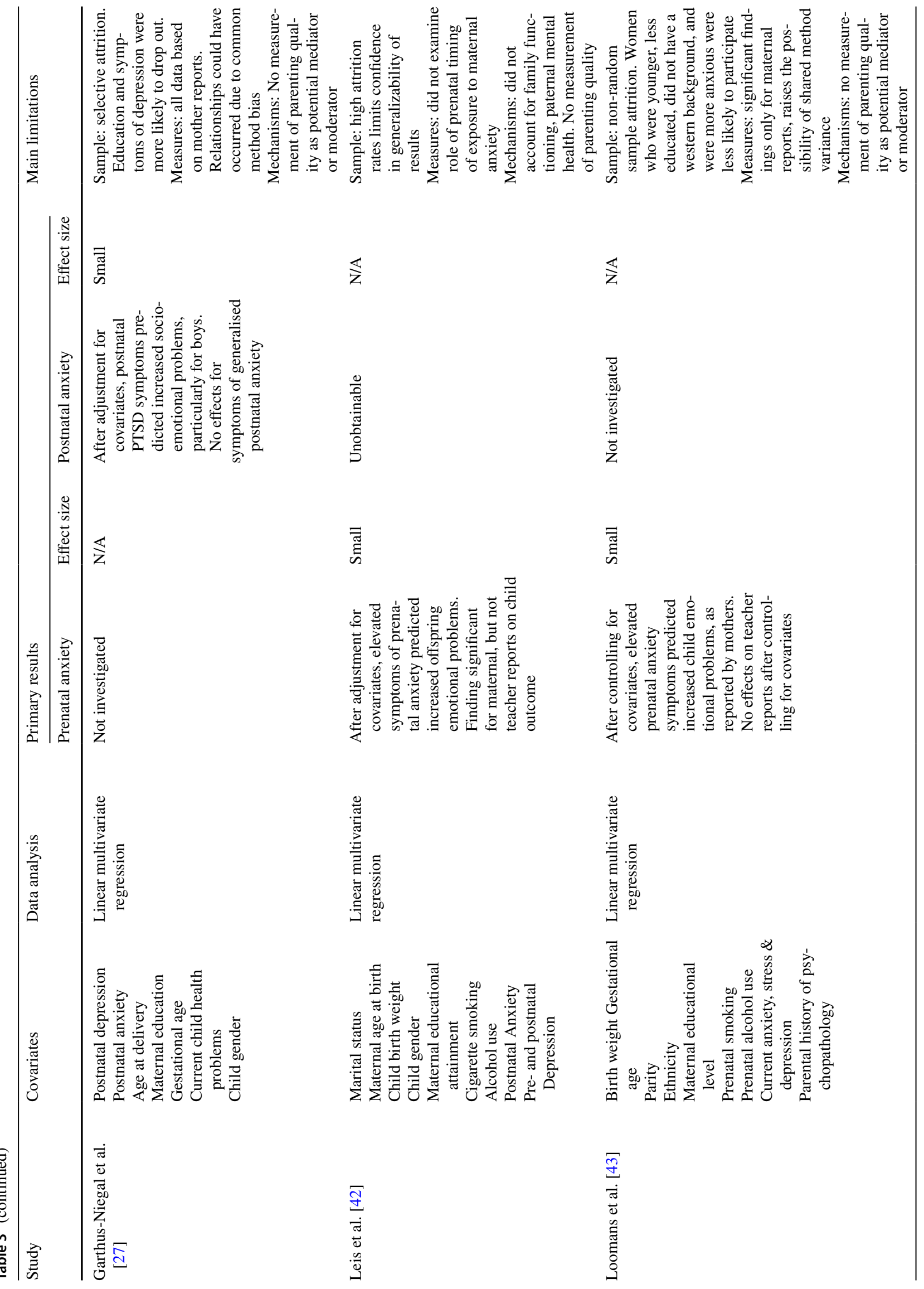




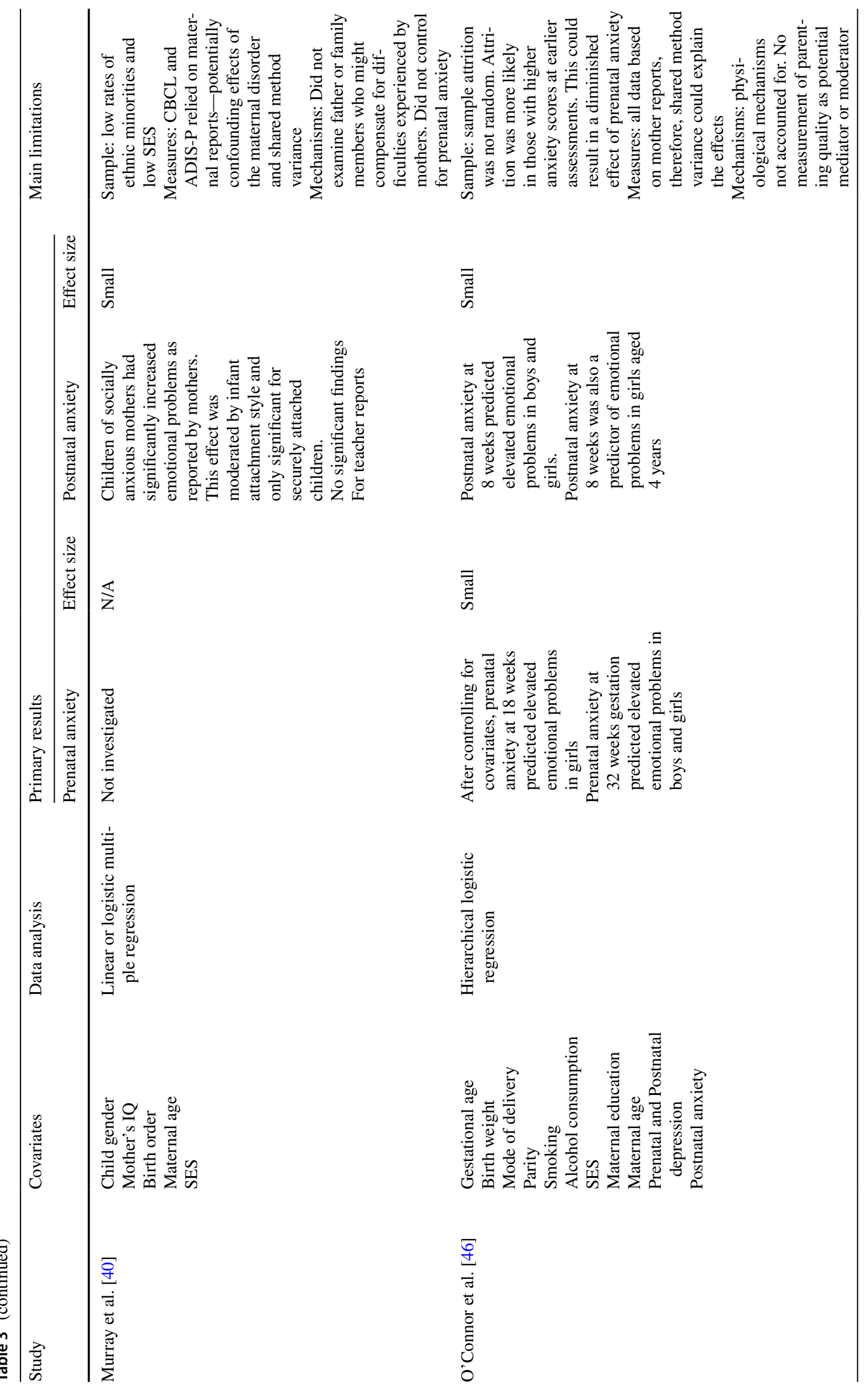




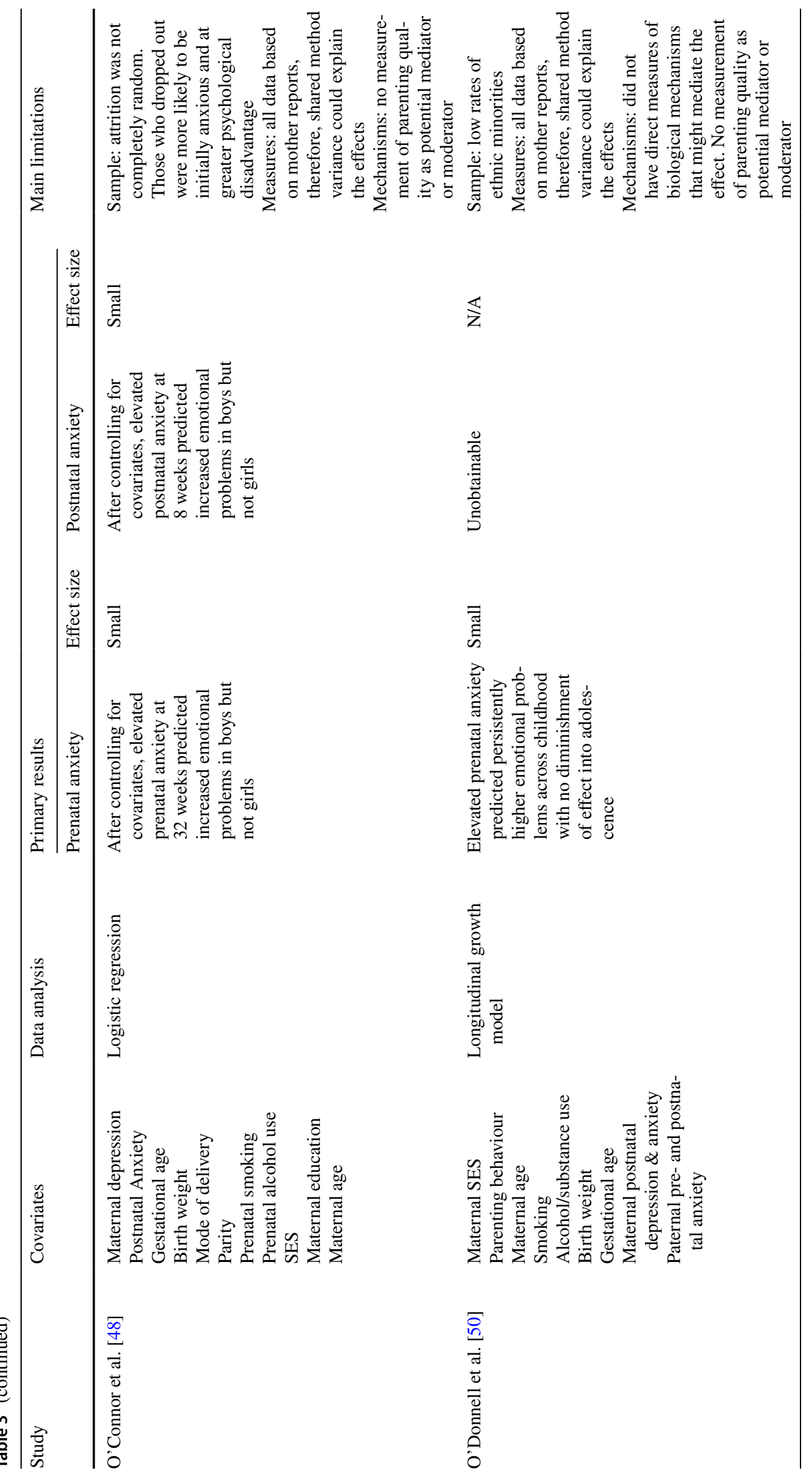




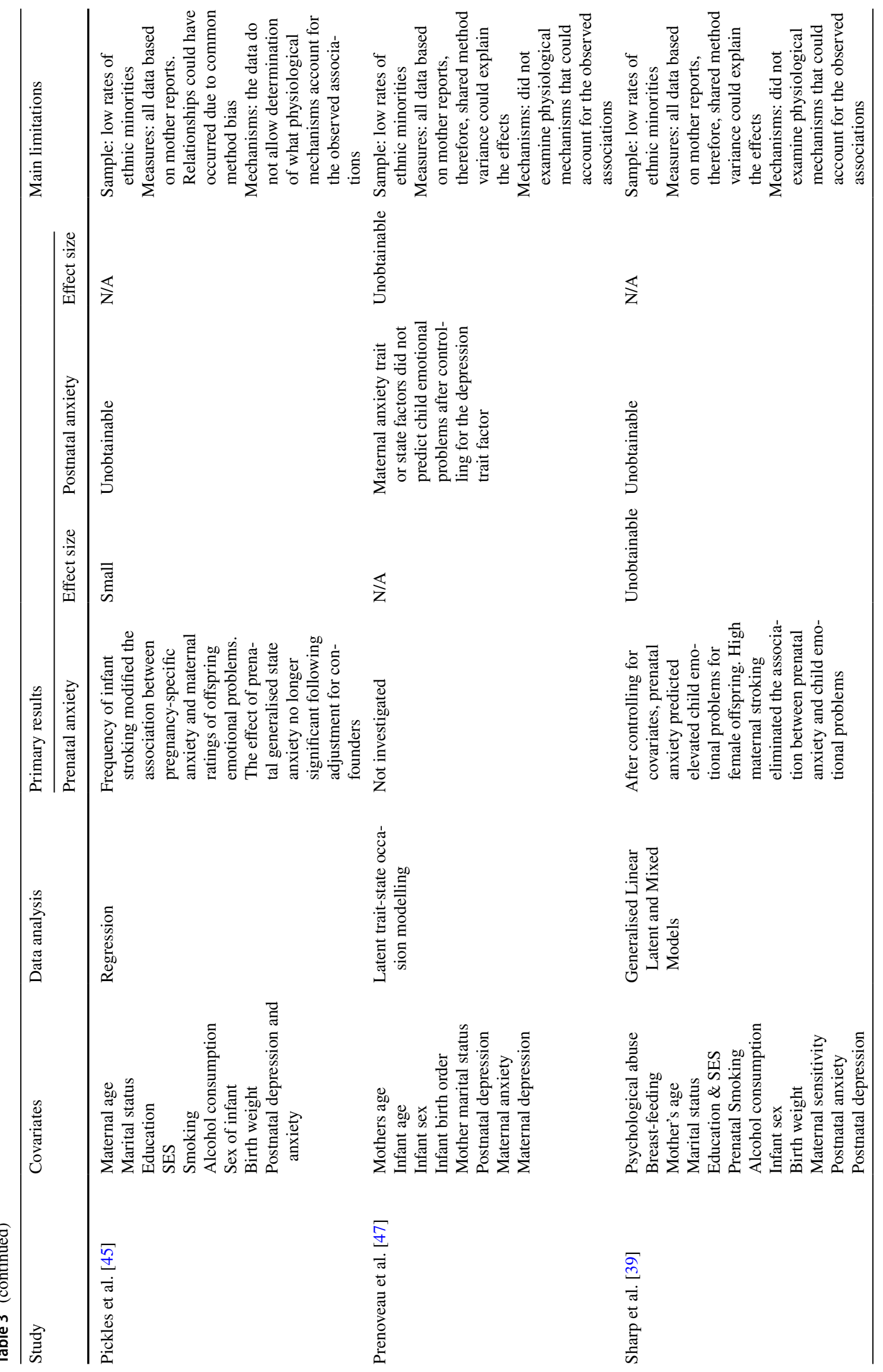




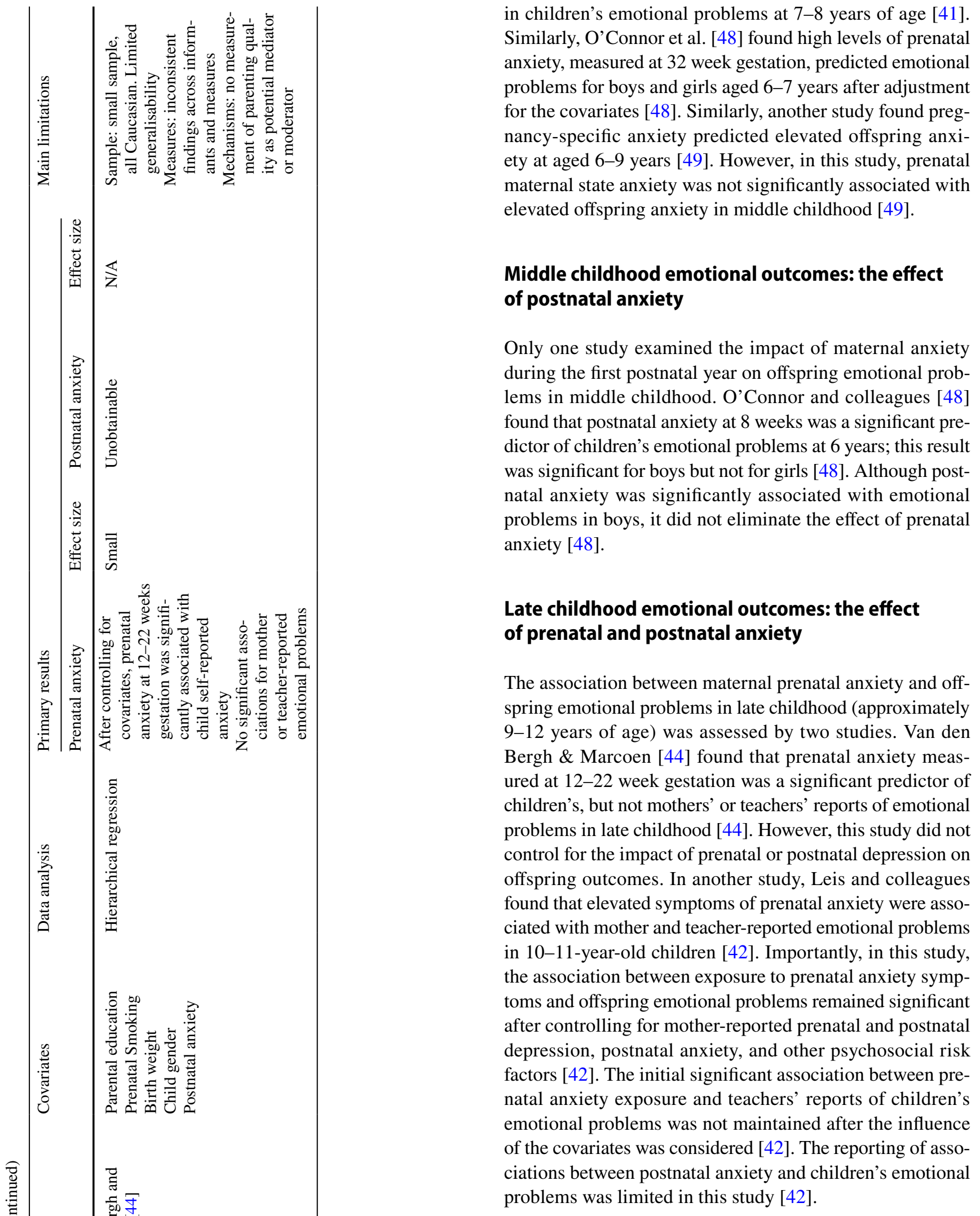




\section{The effects of prenatal anxiety across childhood}

O’Donnell, Glover, Barker \& O'Connor [50] measured children's emotional problems using the SDQ on five occasions from age 4-13 years [50]. The correlations between maternal prenatal anxiety and offspring emotional problems were statistically significant across the 5 timepoints (offspring ages $4,7,9,11.5$, and 13 years). The correlation coefficients were almost identical when prenatal anxiety was measured at both 18 and 32 week gestation-suggesting no/minimal timing effects [50]. In addition, longitudinal growth analyses found that child emotional problems changed over time in a U-shaped manner, with lower scores at age 9 years than at 4 or 13 years.

\section{Maternal prenatal anxiety and children's emotional problems: mechanisms of effect}

Three studies examined potential moderators of the association between maternal prenatal anxiety and child emotional problems. Murray and colleagues [40] found that children of mothers with prenatal social anxiety had higher levels of emotional problems than children of non-anxious controls [40]. When examining whether this relationship was moderated by child attachment style, the effect of maternal social anxiety was significant for securely attached children but not for those who were insecurely attached. Although prenatal anxiety at 20 weeks was found to be predictive of emotional problems at child age 4-5 years, Murray et al. [40] did not control for the effects of prenatal or postnatal depression [40]. Sharp and colleagues [39] hypothesised that maternal stroking would modify the association between prenatal generalised state anxiety and child emotional problems [39]. In this study, with increasing prenatal anxiety, the daughters of low stroking mothers showed increasing emotional problems, whereas this effect was not observed in girls, whose mothers were in the high stroking group, nor was it seen in boys. This study received the highest quality rating. Analyses based on the same cohort by Pickles et al. [45] examined whether the effect of early maternal stroking was still evident 1 year later [45]. After controlling for postnatal anxiety and depression, the frequency of infant stroking modified associations between pregnancy-specific prenatal anxiety, but not generalised prenatal state anxiety, and mothers' ratings of children's emotional problems [45]. There was no indication of a sex difference and the test of the three-way interaction; pregnancy-specific anxiety by stroking by sex of child was non-significant. Despite both these studies being derived from the same cohort and having high-quality ratings, different measures of prenatal anxiety were used and the sample size varies considerably which makes the results difficult to compare.

\section{Discussion}

\section{Summary of findings}

This review systematically evaluated the evidence relating prenatal and postnatal maternal anxiety to children's emotional problems at different phases of development. Based on the findings of the 14 papers which met the inclusion criteria, there was evidence for a small effect of prenatal maternal anxiety [39-44, 46, 48, 50] and pregnancy-specific anxiety $[45,49]$ on child emotional problems following the adjustment for correlated risk factors. There was also preliminary evidence for the impact of postnatal anxiety on child emotional problems [27, 41, 46-48] again representing a small effect following the adjustment for correlated risk factors. However, the low number and overall quality of the studies including postnatal anxiety render this an unreliable conclusion that requires further research. Across the included studies, several methodological weaknesses limit the ability to draw definitive conclusions.

\section{Sample characteristics}

Half the included papers report findings from two longitudinal cohort studies, five from the Avon Longitudinal Study of Parents and Children study [ALSPAC; 51] and two from the Wirral Child Health and Development Study [39, 45]. Therefore, a combination of limited geographical coverage and study attrition due to missing data and exclusion criteria, mean that the findings might have limited generalisability.

\section{Maternal anxiety measurement}

Assessment of maternal anxiety at different stages of preor post-pregnancy makes the studies difficult to compare. All of the studies relied on naturally occurring variations in maternal anxiety in community not clinical populations and the longitudinal designs with large community samples were strengths of most studies. However, selective attrition could mean that studies were examining associations among the less vulnerable individuals. For example, attrition analysis in one study found that mothers who did not provide data at the assessed timepoint were more anxious, younger, less likely to have a university degree and more likely to have smoked in pregnancy [48]. Implications of missing a disproportionate number of children exposed to high levels of anxiety may lead to an underestimation of the long-term effect of more severe perinatal anxiety. 


\section{Mechanisms that mediate or moderate child emotional outcomes}

There is a well-established association between prenatal and postnatal maternal distress, and consequently, prenatal exposures will co-vary with postnatal exposures [3]. Therefore, it needs to be determined whether maternal prenatal anxiety, which is presumed to affect the developing fetus through 'fetal programming', has a significant, unique effect on child outcomes above and beyond known associations, such as parenting behaviours [51,52]. Anxiety and depression symptoms also often co-occur and the presence of co-morbidity is a marker of severity [53]. Inadequate measurement of or control for postnatal maternal anxiety or co-morbid postnatal depression could result in the misattribution of postnatally mediated mechanisms to prenatal biological ones and the majority of studies failed to adequately consider these potential mechanisms.

It is also difficult to separate the effects of maternal perinatal anxiety from the consequence of other factors that might contribute to child emotional problems that were not measured in many of the included studies. For example, the reviewed studies could not rule out potential genetic factors that might affect the observed association. Genetically informed studies involving children conceived through in vitro fertilisation who were not genetically related to their mothers provide strong evidence that the environment contributes to poor child mental health including anxiety risk $[54,55]$. The impact of prenatal exposure to psychotropic medication also needs to be considered. In a recent paper from the Norwegian Mother and Child cohort study investigating the effect of prenatal exposure to selective serotonin reuptake inhibitors (SSRIs), the findings suggest that there was no substantial increased risk for externalizing, emotional, or social problems in preschool-aged children following prenatal SSRI exposure [56]. However, an association between prenatal psychotropic drug exposure and increased rates of depression diagnoses in older offspring has previously been documented [57], so this requires further study.

Only one study included endocrine (cortisol) measures to test for potential underlying mechanisms consistent with the fetal programming hypothesis [48]. In this study, maternal cortisol and psychosocial distress exerted independent effects on child mental health [49]. The other studies in this review did not examine physiological factors. Therefore, direct assessments of the physiological processes that may explain the observed associations and have been implicated by animal and human research (e.g., fetal programming) cannot be determined by this review. Similarly, only eight studies examined gender differences with very mixed results, so the impact of gender on the association between maternal perinatal anxiety and child emotional outcomes is inconclusive.

\section{Shared method variance}

All studies had methodological issues related to shared method variance, which reduced their quality ratings. The use of self-report questionnaires meant that often mothers were reporting on their own levels of anxiety and also on their perceptions of their child's behaviour. This could lead to mothers with elevated symptoms of anxiety and depression over- or misreporting their child's emotions and mothers who do not experience anxiety not recognising symptoms in their children. Across the included studies, the effect of maternal anxiety on child emotional problems was most profound when mothers had reported on their child's behaviour. However, only a minority of studies used multiple informants on the child outcome measure, for example, fathers [38, 41] or teachers [42, 43, and 44]. Murray et al. [40] and Prenoveau et al. [47] were the only studies to use observational measures of child outcomes. In addition to the effect of shared method variance, there is also the impact of context on understanding and interpreting children's behaviours and emotional experience: for example, the parents knowing their child across multiple settings compared to teachers who in turn will be more able to view a child's behaviour and emotional experience in comparison with peers $[58,59]$. Nevertheless, it is likely that shared method variance partly explains the greater associations when mothers report on their own anxiety symptoms and their child's emotional problems.

\section{Implications for future research}

Future studies should incorporate multiple informants and employ a range of methods to measure children's emotional problems (e.g., standardised clinical interviews, bio-markers of child emotional problems, and observational data). Biological measures would enable future studies to examine the association between both biological and psychological factors during gestation and the risk for adverse emotional outcomes in childhood. Similarly, the inclusion of postnatal risk factors such as quality of parenting should be incorporated as a potential mediator or moderator of the association between perinatal anxiety and children's emotional problems. Future research should also examine the role of timing of exposure to prenatal anxiety (e.g., number of week gestation) as well as clinically significant levels of maternal anxiety as identified through diagnostic interviews.

\section{Implications for clinical practice}

The finding that maternal anxiety during the perinatal period was associated with adverse emotional outcomes in children provides support for a preventative approach to infant developmental problems. Historically, much attention has 
been paid to postnatal depression, which has focused its approach on prevention and intervention beginning during the postnatal period. This review has demonstrated that anxiety experienced during the prenatal period has significant consequences for a child's emotional development, highlighting the need for prenatal interventions for maternal mental health. It is possible that addressing maternal mental health, including anxiety in pregnancy, may in turn affect the mother's relationship with her child and the overall family functioning, with widespread effects. These results were found in community-based samples, suggesting that mental health assessment and intervention are important components of routine perinatal care. This review provides health and economic-related arguments to support increased screening and access to specialist perinatal mental health services, with long-term implications for women's mental health, child development, and well-being.

\section{Strengths and limitations of the current review}

The findings of this review are the result of a thorough, systematic process reviewing a large number of articles. The inclusion of studies that measured anxiety symptoms alongside those that measured clinical diagnoses of anxiety is a strength of the review. The results highlight the importance of measurement of different forms of anxiety when considering the impact on child emotional problems. Despite the strengths of this review, some limitations exist. Only articles published in English were included, which means that relevant articles published in other languages may have been overlooked. It is also acknowledged that the use of a quality assessment tool involves a degree of subjectivity in the ratings process. Furthermore, inconsistent presentation of the results across the included studies rendered a meta-analysis difficult and beyond the scope of the current paper.

\section{Conclusion}

There is some evidence that prenatal and postnatal anxiety exposure may lead to adverse emotional outcomes in children, but further evidence is needed. Expanding on the literature and improving the methodological rigour of such studies will enable a better understanding of the effects of maternal anxiety during the perinatal period on child emotional outcomes. Such research could lead to the improved identification of at-risk parents and children with appropriate opportunities for intervention and prevention.

\section{Compliance with ethical standard}

Conflict of interest On behalf of all authors, the corresponding author states that there is no conflict of interest.
Open Access This article is distributed under the terms of the Creative Commons Attribution 4.0 International License (http://creativeco mmons.org/licenses/by/4.0/), which permits unrestricted use, distribution, and reproduction in any medium, provided you give appropriate credit to the original author(s) and the source, provide a link to the Creative Commons license, and indicate if changes were made.

\section{References}

1. National Institute for Health and Care Excellence. Prenatal and Postnatal Mental Health: Clinical Management and Service Guidance. NICE; 2014

2. Wenzel A, Haugen E, Jackson L, Robinson K (2003) Prevalence of generalized anxiety at eight weeks postpartum. Arch Women's Mental Health. 6(1):43-49

3. Heron J, O'Connor T, Evans J, Golding J, Glover V (2004) The course of anxiety and depression through pregnancy and the postpartum in a community sample. J Affect Disord 80(1):65-73

4. Lydsdottir L, Howard L, Olafsdottir H, Thome M, Tyrfingsson P, Sigurdsson J (2014) The mental health characteristics of pregnant women with depressive symptoms identified by the Edinburgh postnatal depression scale. J Clin Psychiatry 75(04):393-398

5. Wisner K, Sit D, McShea M, Rizzo D, Zoretich R, Hughes C et al (2013) Onset timing, thoughts of self-harm, and diagnoses in postpartum women with screen-positive depression findings. JAMA Psychiatry 70(5):490

6. Waters CS, Hay D, Simmonds J, van Goozen S (2014) Prenatal depression and children's developmental outcomes: potential mechanisms and treatment options. Eur Child Adolesc Psychiatry 23(10):957-971

7. Bauer A, Parsonage M, Knapp M, Lemmi V, Adelaja B (2014) The costs of perinatal mental health problems. Centre for Mental Health and London School of Economics, London

8. Glasheen C, Richardson G, Fabio A (2009) A systematic review of the effects of postnatal maternal anxiety on children. Arch Women's Mental Health 13(1):61-74

9. Rahman A, Surkan P, Cayetano C, Rwagatare P, Dickson K (2013) Grand challenges: integrating maternal mental health into maternal and child health programmes. PLoS Med 10(5):e1001442

10. Sharp D, Hay D, Pawlby S, Schmücker G, Allen H, Kumar R (1995) The impact of postnatal depression on boys' intellectual development. J Child Psychol Psychiatry 36(8):1315-1336

11. Ramchandani P, Stein A, Evans J, O'Connor T (2005) Paternal depression in the postnatal period and child development: a prospective population study. Lancet 365(9478):2201-2205

12. Murray L, Arteche A, Fearon P, Halligan S, Croudace T, Cooper P (2010) The effects of maternal postnatal depression and child sex on academic performance at age 16 years: a developmental approach. J Child Psychol Psychiatry 51(10):1150-1159

13. Murray L (2009) The development of children of postnatally depressed mothers: evidence from the Cambridge longitudinal study. Psychoanal Psychother 23(3):185-199

14. O'Connor T, Monk C, Fitelson E (2014) Practitioner review: maternal mood in pregnancy and child development-implications for child psychology and psychiatry. J Child Psychol Psychiatry 55(2):99-111

15. Zahn-Waxler C, Klimes-Dougan B, Slattery M (2000) Internalizing problems of childhood and adolescence: prospects, pitfalls, and progress in understanding the development of anxiety and depression. Dev Psychopathol 12(3):443-466

16. Moher D, Liberati A, Tetzlaff J, Altman D (2009) Preferred reporting items for systematic reviews and meta-analyses: the PRISMA statement. BMJ. 339(jul21 1):b2535-b2535 
17. Liberati A, Altman D, Tetzlaff J, Mulrow C, Gotzsche P, Ioannidis J et al (2009) The PRISMA statement for reporting systematic reviews and meta-analyses of studies that evaluate health care interventions: explanation and elaboration. PLoS Med 6(7):e1000100

18. Covidence-Accelerate your systematic review [Internet]. Covidence.org. 2017 [cited 3 March 2017]. Available from: https:// www.covidence.org

19. Cohen J (1988) Statistical power analysis for the behavioral sciences. Lawrence Erlbaum Associates, Hillsdale

20. Steiger JH (2004) Beyond the F test: effect size confidence intervals and tests of close fit in the analysis of variance and contrast analysis. Psychol Methods 9(2):164-182

21. Nieminen P, Lehtiniemi H, Vähäkangas K, Huusko A, Raution A (2013) Standardised regression co-efficient as an effect size index in summarising findings in epidemiological studies. Epidemiol Biostat Publ Health 10(4):e8854-15

22. Speilberger C (1977) State-Trait Anxiety Inventory: self-evaluation questionnaire, 1st edn. Consulting Psychol, Palo Alto

23. Spielberger C, Gorsuch R, Lushene R, Vagg P, Jacobs G (1983) Manual for the state-trait anxiety inventory, 1st edn. Consulting Psychologists Press, Palo Alto

24. Barnes L, Harp D, Jung W (2002) Reliability Generalization of Scores on the Spielberger State-Trait Anxiety Inventory. Educ Psychol Meas 62(4):603-618

25. Crown S, Crisp A (1966) A, short clinical diagnostic self-rating scale for psychoneurotic patients: the Middlesex Hospital Questionnaire (M.H.Q.). The British Journal of Psychiatry. 112(490):917-923

26. Elliott S, Leverton T, Sanjack M, Turner H, Cowmeadow P, Hopkins J et al (2000) Promoting mental health after childbirth: a controlled trial of primary prevention of postnatal depression. $\mathrm{Br}$ J Clin Psychol 39(3):223-241

27. Garthus-Niegel S, Ayers S, Martini J, von Soest T, Eberhard-Gran M (2016) The impact of postpartum post-traumatic stress disorder symptoms on child development: a population-based, 2-year follow-up study. Psychol Med 47(01):161-170

28. First M, Spitzer R, Gibbon M, Williams J (1995) Structured Clinical Interview for DSM-IV Axis I diagnoses, 1st edn. New York State Psychiatric Institute, Biometrics Research Department, New York

29. Achenbach T, Rescorla L (2001) Manual for the ASEBA schoolage forms and profiles: An integrated system of multi-informant assessment, 1st edn. University of Vermont, research center for children youth \& families, Burlington (VT)

30. Achenbach T (1991) Manual for the child behaviour checklist 4-18 and 1991 profile, 1st edn. University of Vermont, Department of Psychiatry, Burlington

31. Achenbach T (1991) Manual for the Teacher's report form and 1991 profile, 1st edn. University of Vermont, Department of Psychiatry, Burlington

32. Goodman R, Scott S (1999) Comparing the strengths and difficulties questionnaire and the child behaviour checklist: is small beautiful? J Abnorm Child Psychol 27:17-24

33. Elander J, Rutter M (1996) Use and development of the Rutter parents' and teachers' rating scales. Int J Methods Psychiatric Res 6(2):63-78

34. Squires J, Bricker D, Twombly E (2005) The ASQ: SE User's guide: for the Ages \& Stages Questionnaires, Social-Emotional: a parent-completed, child-monitoring program for social-emotional behaviours. 1st ed. Baltimore, MD: Paul H. Brookes Pub. Co

35. Meltzer H, Gatward R, Goodman R, Ford F (2000) The mental health of children and adolescents in Great Britain, 1st edn. Stationery Office, London

36. Silverman W, Saavedra L, Pina A (2001) Test-retest reliability of anxiety symptoms and diagnoses with the anxiety disorders interview schedule for DSM-IV: child and parent versions. J Am Acad Child Adolesc Psychiatry 40(8):937-944

37. Bakker F, Van Wieringen $P$, Van der Ploeg $H$, Spielberger $C$ (1989) Manual for the self-evaluation questionnaire for children, STAIC, 1st edn. Swets \& Zeitlinger, Lisse

38. de Bruijn A, van Bakel H, van Baar A (2009) Sex differences in the relation between prenatal maternal emotional complaints and child outcome. Early Human Dev 85(5):319-324

39. Sharp H, Hill J, Hellier J, Pickles A (2014) Maternal prenatal anxiety, postnatal stroking and emotional problems in children: outcomes predicted from pre- and postnatal programming hypotheses. Psychol Med 45(02):269-283

40. Murray L, Pella J, De Pascalis L, Arteche A, Pass L, Percy R et al (2014) Socially anxious mothers' narratives to their children and their relation to child representations and adjustment. Dev Psychopathol 26(4pt2):1531-1546

41. Barker E, Jaffee S, Uher R, Maughan B (2011) The contribution of prenatal and postnatal maternal anxiety and depression to child maladjustment. Depression Anxiety. 28(8):696-702

42. Leis J, Heron J, Stuart E, Mendelson T (2014) Associations between maternal mental health and child emotional and behavioural problems: does prenatal mental health matter? J Abnorm Child Psychol 42(1):161-171

43. Loomans E, der Stelt O, van Eijsden M, Gemke R, Vrijkotte T, den Bergh B (2011) Prenatal maternal anxiety is associated with problem behaviour at age five. Early Hum Dev 87(8):565-570

44. Van den Bergh B, Marcoen A (2004) High prenatal maternal anxiety is related to ADHD symptoms, externalizing problems, and anxiety in 8- and 9-year-olds. Child Dev 75(4):1085-1097

45. Pickles A, Sharp H, Hellier J, Hill J (2016) Prenatal anxiety, maternal stroking in infancy, and symptoms of emotional and behavioural disorders at 3.5 years. Eur Child Adolesc Psychiatry 26(3):325-334

46. O'Connor T, Heron J, Golding J, Beveridge M, Glover V (2002) Maternal prenatal anxiety and children's behavioural/emotional problems at 4 years: report from the Avon Longitudinal Study of Parents and Children. Br J Psychiatry 180(6):502-508

47. Prenoveau J, Craske M, West V, Giannakakis A, Zioga M, Lehtonen A et al (2017) Maternal postnatal depression and anxiety and their association with child emotional negativity and behaviour problems at two years. Dev Psychol 53(1):50-62

48. O'Connor T, Heron J, Golding J, Glover V (2003) Maternal prenatal anxiety and behavioural/emotional problems in children: a test of a programming hypothesis. J Child Psychol Psychiatry 44(7):1025-1036

49. Davis E, Sandman C (2012) Prenatal psychobiological predictors of anxiety risk in preadolescent children. Psychoneuroendocrinology. 37(8):1224-1233

50. O'Donnell K, Glover V, Barker E, O'Connor T (2014) The persisting effect of maternal mood in pregnancy on childhood psychopathology. Dev Psychopathol 26(02):393-403

51. Golding J, Pembrey M, Jones R (2001) The ALSPAC Study Team. ALSPAC-The Avon Longitudinal Study of Parents and Children. Paediatr Perinat Epidemiol 15(1):74-87

52. DiPietro J (2012) Maternal stress in pregnancy: considerations for fetal development. J Adolesc Health 51(2):S3-S8

53. Andrews G, Sanderson K, Slade T, Issakidis C (2000) Why does the burden of disease persist? Relating the burden of anxiety and depression to effectiveness of treatment. Aust N Z J Psychiatry 34(s1):A3-A3

54. Rice F, Harold G, Boivin J, van den Bree M, Hay D, Thapar A (2010) The links between prenatal stress and offspring development and psychopathology: disentangling environmental and inherited influences. Psychol Med 40(02):335-345

55. Lewis G, Rice F, Harold G, Collishaw S, Thapar A (2011) Investigating environmental links between parent depression and 
child depressive/anxiety symptoms using an assisted conception design. J Am Acad Child Adolescent Psychiatry 50(5):451-459. e1

56. Lupattelli A, Wood M, Ystrom E, Skurtveit S, Handal M, Nordeng $\mathrm{H}$ (2018) Effect of time-dependent selective serotonin reuptake inhibitor antidepressants during pregnancy on behavioural, emotional, and social development in preschool-aged children. J Am Acad Child Adolesc Psychiatry 57(3):200-208

57. Malm H, Brown AS, Gissler M, Gyllenberg D, Hinkka-YliSalomaki S, McKeague IW et al (2016) Gestational exposure to selective serotonin reuptake inhibitors and offspring psychiatric disorders: a national register-based study. J Am Acad Child Adolescent Psychiatry. 55(5):359-366

58. Briggs-Gowan M, Carter A, Schwab-Stone M (1996) Discrepancies among mother, child, and teacher reports: examining the contributions of maternal depression and anxiety. J Abnorm Child Psychol 24(6):749-765

59. Youngstrom E, Loeber R, Stouthamer-Loeber M (2000) Patterns and correlates of agreement between parent, teacher, and male adolescent ratings of externalizing and internalizing problems. J Consult Clin Psychol 68(6):1038-1050 